\title{
Time Series Regression Model for Prediction Of Closing Values of the Stock using an Adaptive NARX Neural Network
}

\author{
Saurabh Labde \\ Undergraduate in Electronics \& \\ Telecommunication \\ Engineering from D.J Sanghvi \\ College of engineering
}

\author{
Stuti Patel \\ Undergraduate in Electronics \& \\ Telecommunication \\ Engineering from D.J Sanghvi \\ College of engineering
}

\author{
Megh Shukla \\ Undergraduate in Electronics \& \\ Telecommunication \\ Engineering from D.J Sanghvi \\ College of engineering
}

\begin{abstract}
In the financial sector, the dependence on modern technologies has seen an increase since the last two decades. The advancements in the field of neural networks and machine learning has provided a number of financial tools. These tools often seen to form the basis of financial computations such as stock market prediction, bankruptcy prediction, risk assessment etc. In this paper, we propose a model based on an adaptive NARX neural network to predict the closing price of any stock. This is a non-linear auto regressive exogenous input model which uses delays in the input as well as the output acting as memory slots thereby increasing the accuracy of the prediction. This model uses a time series approach to analyze and predict the closing price . This NARX model is trained using three input values - the opening price of the stock, the highest price of the stock and the lowest price of the stock for the day. The target values are also fed to the network as it is a supervised learning model. Levenberg-marquardt algorithm has been used for training the network. The accuracy of the network is determined with the help of the mean squared error. In this model, we have made use of a closed loop with reduced input delays as well as a closed loop model for making predictions and the accuracy of each case is determined to analyze the working of a NARX neural network and to determine the optimum configuration.
\end{abstract}

\section{Keywords}

Neural Networks, NARX ( Non-Linear Auto Regressive Exogenous inputs model), Training data, Target data, delays, mean - squared error.

\section{INTRODUCTION}

In today's world, stock market is a widely growing phenomenon. Everyone, may it be a layman or a financial expert invests in stocks. In such a case, the use of modern technology such as neural networks can be used to enhance this experience and provide insights into the process. Neural network models are developed to accurately predict the closing prices of the stocks, which serves as an aid to the common investors. On a large scale, this technology is commonly used by the giants in the field to predict the values of the stocks which form the basis for their decisions. Thus the stock prediction models are imperative in making million dollar decisions and the accuracy of such a model cannot be compromised. In this paper, a model is proposed based on NARX neural network to predict the closing price of any stock with high accuracy. This model is based on a time series approach which plots the stock values as a continuous function of time and monitors the changes constantly and thus enables us to train the network efficiently to obtain a greater accuracy for its prediction. For the stock of any company, there are a number of factors that affects its values. The amalgam of these values determine the closing value of the stock. As it is extremely difficult to account for all the factors , we have used 3 parameters which provide the best possible result for the predictions. Thus the opening prices of the stock , highest value of the stock in the day and the lowest values are used to train the net. This model makes use of the Levenberg-marquardt algorithm to train the neural net and the mean squared errors are computed to determine the accuracy of the model. The NARX model uses the input and output delays as memory taps used to train the network for the past values to get the optimum results. We have made use of the closed loop as well as the closed loop with reduced input delays calculations and determined the result in each case to learn about the optimum configuration to be used.

\section{CURRENT MODEL}

The current neural net models for stock value prediction makes use of the feed forward multi layered perceptron or back propagation algorithms to train the network. Thus, this model cannot adequately account for the volatility of the stock and thus provides a prediction with a lower accuracy. With such a neural network the training of the network is faster, however the results sometimes show a greater deviation from the target.

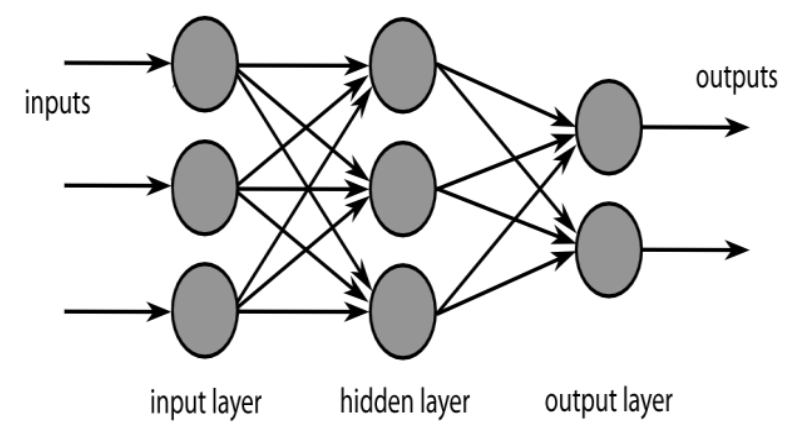

Fig 1. Current neural network model architecture

\section{PROPOSED NEURAL NETWORK MODEL ARCHITECTURE}

To overcome the drawbacks of the current models, in this project we have made use of a Non-linear Auto Regressive Neural Network with exogenous inputs (NARX). A NARX model is a recurrent type of dynamic network. Here the output 
of the network is a function of the delayed input and output values as can be shown below in the equation:-

$\mathrm{yp}(\mathrm{t})=\mathrm{f}(\mathrm{y}(\mathrm{t}-1), \mathrm{y}(\mathrm{t}-2), \mathrm{y}(\mathrm{t}-3), \ldots, \mathrm{u}(\mathrm{t}), \mathrm{u}(\mathrm{t}-1), \mathrm{u}(\mathrm{t}-2), .$.

where the next value of the dependent output signal $y(t)$ is regressed on previous values of the output signal and the previous values of an independent (exogenous) input signal. The basic structure of a NARX network can be modelled as below:

\section{Nonlinearity Estimator}

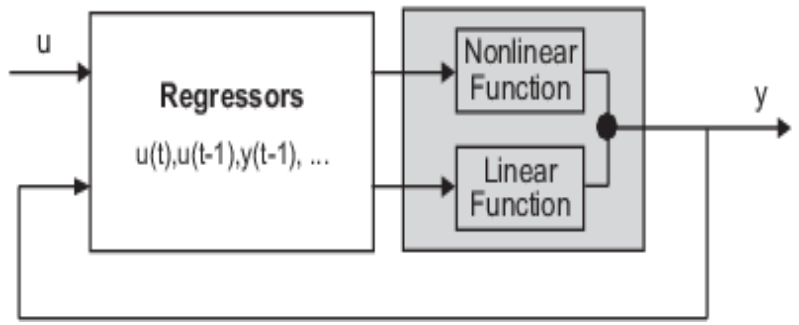

Fig 2. Basic NARX neural network structure

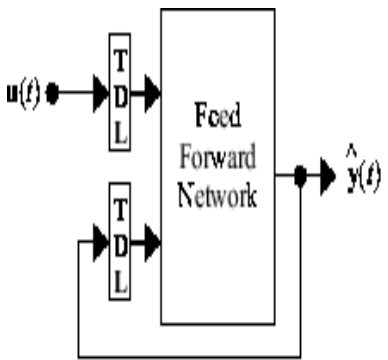

Paralkel Architecture

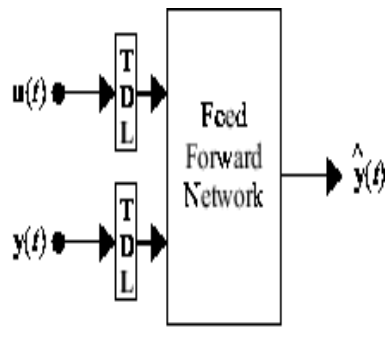

Series-Parallel Architecture

\section{Fig 3. Parallel and Series-Parallel architecture of NARX}

$\mathrm{u}(\mathrm{k})$ represents the past exogenous values (trend of the past stock values throughout the day)

$y(k)$ represents the past values of the actual series to be predicted(closing prices).

$\hat{y}(k)$ indicates the predicted values.

Here we have used a parallel architecture . A non-linear model is advantageous to use as compared to a linear model as it can estimate the non-linearities with which a stock price changes daily. The linear model captures the rising and settling behavior in the time series prediction but does not account for sharp variations at 90 degrees. Whereas a non-linear model can take into account the non-linearities which help in overall improvement of the model and helps in a smoother curve fitting for the data. The feedback taps included in the architecture of the network act as memory storages which stores the next input while the previous inputs are being used for training the network.

\section{DATA GENERATION}

We have generated the data of various stocks to be used from Yahoo Finance India records of stocks i.e. https://in.finance.yahoo.com/ website. The historical data of the stocks used to test the neural network are available in the form of a downloadable spreadsheet. We have generated data for an approximate of 2 years from 1st January, 2012 upto 1st January, 2015.The neural network is trained using this data across two years. Out of all the available samples for a particular stock $60 \%$ forms the training data, $5 \%$ forms the testing data and the remaining $35 \%$ forms the testing dataset. The output of the network gives us a prediction of the closing price of the stock for the next day i.e. for 2nd January, 2015.

\section{IMPLEMENTATION}

Here we have performed time series modeling of the historical record of a stock using a nonlinear auto regressive exogenous model (NARX). The model relates the current value of a time series where one would like to explain or predict to both: past values of the same series; and current and past values of the driving (exogenous) series. The network used is dynamic in nature as it has feedback loops (outputs of a neuron fed back to some previous neuron) and taps (delay lines that feed the network with past values of inputs). The use of a NARX network helps to increase the accuracy of prediction as well as can be used for real time prediction of stock values.

The model proposed here has three sets of exogenous inputs which are

1) Opening Value of the stock.

2) Highest price of the stock.

3) Lowest price of the stock during a particular day.

The target series comprises of the closing price of the stock daily. The output of the neural network is predicting closing prices over the entire target series along with the next value in the series. This is a curve-fitting problem which is implemented using MATLAB version 2015a. A parallel NARX network is created using "narxnet" command which helps in increasing the accuracy of the network .Also here we have plotted the time series model of a modified NARX network which is comprised of fewer input delays and greater output delays. Hence the response obtained can be used for further time series analysis. Algorithm used for training of the network is "Levenberg-Marquardt".It is an iterative method which is a popular alternative to the Gauss-Newton method of finding the minimum of a function that is a sum of squares of nonlinear functions,

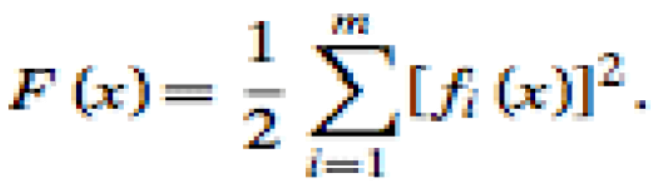

The Levenberg-Marquardt method searches in the direction given by the solution to the equations

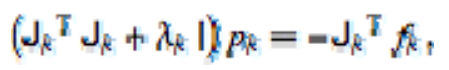

where $\lambda_{\mathrm{K}}$ are non negative scalars and I is the identity matrix. LM algorithm is a combination of Gauss-Newton and Gradient Descent algorithm and hence is more robust as it provides a nice compromise between the speed of Newton's method and the guaranteed convergence of steepest descent.

For Gauss-Newton Method:-

$\nabla t=-[J T(t) J(t)]-1 J(t) e(t)$

For Levenberg-Marqardt Algorithm as a variation of GaussNewton Method we use the following equation for time difference:-

$\nabla \mathrm{t}=-[\mathrm{JT}(\mathrm{t}) \mathrm{J}(\mathrm{t})+\mathrm{uI}]-1 \mathrm{~J}(\mathrm{t}) \mathrm{e}(\mathrm{t})$

We carry out updation of initial weights $w(k)$ using the Jacobian matrix and individual error of different vectors $\mathrm{w}(\mathrm{k}+1)=\mathrm{w}\{-[\mathrm{JT}(\mathrm{t}) \mathrm{J}(\mathrm{t})+\mathrm{uI}]-1 \mathrm{~J}(\mathrm{t}) \mathrm{e}(\mathrm{t})\}$

$\nabla \mathrm{E}(\mathrm{k})=\mathrm{JT}(\mathrm{t}) \mathrm{e}(\mathrm{t})$

$\nabla^{2} \mathrm{E}(\mathrm{k})=\mathrm{JT}(\mathrm{t}) \mathrm{J}(\mathrm{t})+\mathrm{S}(\mathrm{t})$ 
where $\nabla \mathrm{E}(\mathrm{k})$ is the gradient $\nabla^{2} \mathrm{E}(\mathrm{k})$ is the Hessian Matrix of $\mathrm{E}(\mathrm{k})$

$\mathrm{S}(\mathrm{t})=\sum \mathrm{N}$ i=1 ei(t) $\nabla 2$ ei(t) Where $\mathrm{S}(\mathrm{t})$ is the summation of square errors of $\mathrm{N}$ input vectors.

The proposed model of the NARX Neural network has an input dimensionality of 3 , output dimensionality of 1 , number of hidden layer neurons are 10 and the network is being trained for 15 epochs.

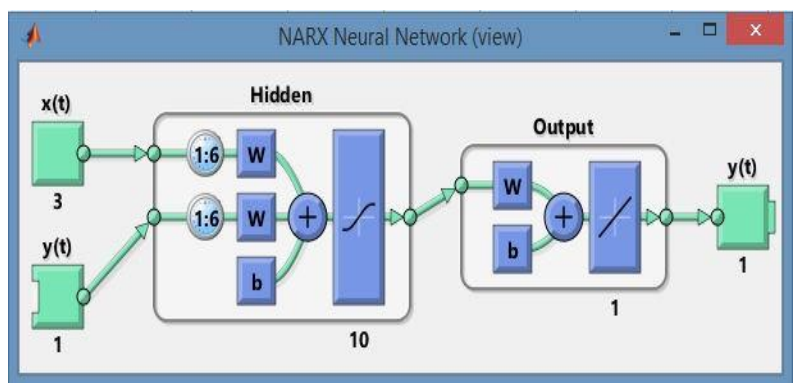

Fig 4. Proposed architecture of the NARX model

The flowchart of the implementation of the NARX net model is shown below:

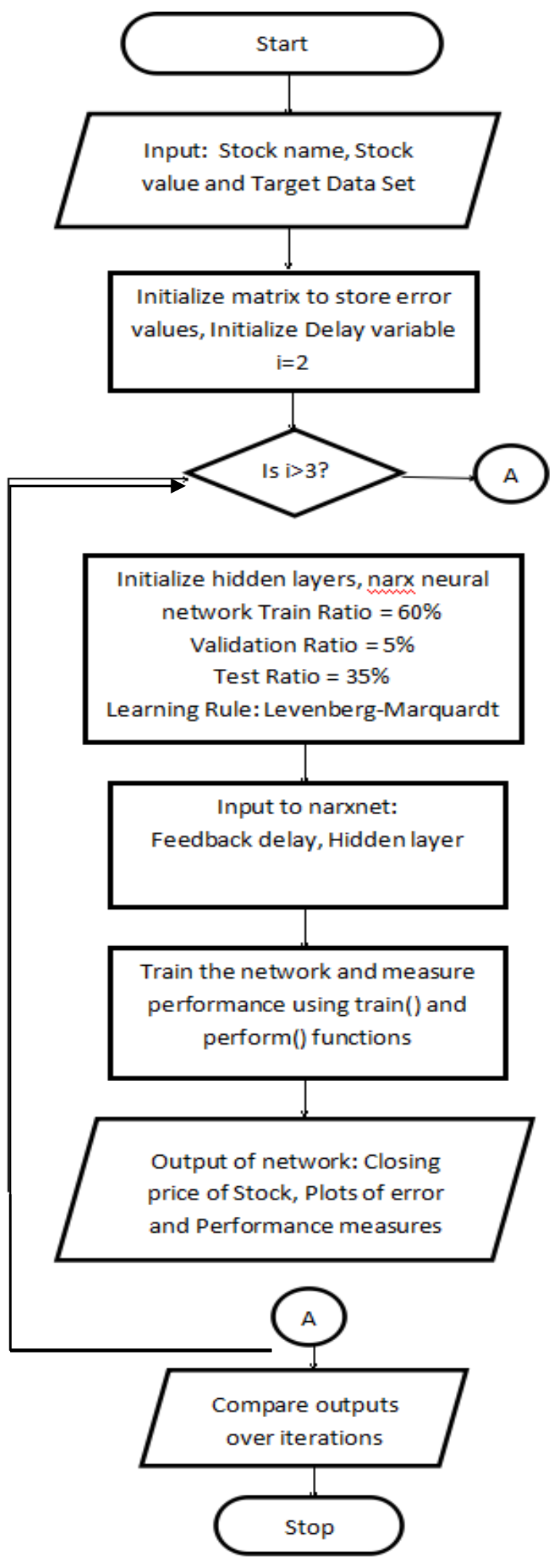

Fig 5. Flowchart of the implementation

\section{RESULTS}

The NARX net model was trained with a data set of 2 years for different stocks. To analyze the working of the new neural network model for different conditions, we have divided the stocks into three distinct classes - First is the stable stocks. 
The stocks in this class do not show a major deviation in its value and hence can be considered as stable. The second class is moderately stable stocks. These stocks show minor deviations in the stock values over the time range. The third class of stocks are the relatively unstable stocks. These stocks have shown a sharp deviation in its values over the given time range and are known to fluctuate. The new model for neural network was tested for the stocks falling under these three classes and its performance was analyzed for the same to help us determine the optimum conditions for the neural network.

Table 1:The table for the values obtained with the neural network for stable stocks is shown below:

\begin{tabular}{|c|c|c|c|}
\hline $\begin{array}{c}\text { Name of the } \\
\text { Stock }\end{array}$ & $\begin{array}{l}\text { Predicted } \\
\text { Price (Rs) }\end{array}$ & $\begin{array}{l}\text { Relative } \\
\text { Mean } \\
\text { Square } \\
\text { Error }\end{array}$ & $\begin{array}{c}\text { Actual } \\
\text { Error }\end{array}$ \\
\hline Accenture & 88.1974 & 0.0103 & 0.0124 \\
\hline MRF & 36975 & 72.39 & 0.026 \\
\hline Reliance Power & 61.5290 & 0.0235 & 0.014 \\
\hline Bharti Airtel & 351.9947 & 0.0772 & 0.0302 \\
\hline Wipro & 546.5847 & 0.0942 & 0.0103 \\
\hline Indiabulls & 67.9554 & 0.0245 & 0.0123 \\
\hline Idea cellular & 155.7477 & 0.0282 & 0.0223 \\
\hline BPCL & 642.9564 & 0.1627 & 0.0166 \\
\hline
\end{tabular}

Table 2:The table for the values obtained with the neural network for moderately stable stocks is shown below:

\begin{tabular}{|c|c|c|c|}
\hline $\begin{array}{c}\text { Name of the } \\
\text { Stock }\end{array}$ & $\begin{array}{l}\text { Predicted } \\
\text { Price (Rs) }\end{array}$ & $\begin{array}{l}\text { Relative } \\
\text { Mean } \\
\text { Square } \\
\text { Error }\end{array}$ & $\begin{array}{c}\text { Actual } \\
\text { Error }\end{array}$ \\
\hline TCS & 2534.1 & 0.607 & 0.0055 \\
\hline $\begin{array}{c}\text { KEC } \\
\text { International }\end{array}$ & 95.4191 & 0.0575 & 0.0076 \\
\hline RadicoKhaitan & 87.5506 & 0.0272 & 0.0029 \\
\hline Infosys & 1092.4 & 2.5208 & 0.0054 \\
\hline ICICI Bank & 240.8923 & 0.5312 & 0.045 \\
\hline ITC & 365.9283 & 0.0467 & 0.0031 \\
\hline $\begin{array}{c}\text { JK tyre \& } \\
\text { industries }\end{array}$ & 131.0568 & 1.4108 & 0.0086 \\
\hline $\begin{array}{c}\text { Reliance } \\
\text { Communications }\end{array}$ & 78.7213 & 0.024 & 0.0098 \\
\hline
\end{tabular}

Table 3:The table for the values obtained with the neural network for moderately stable stocks is shown below:

\begin{tabular}{|c|c|l|l|}
\hline Name of the Stock & $\begin{array}{l}\text { Predicted } \\
\text { Price (Rs) }\end{array}$ & $\begin{array}{l}\text { Relative } \\
\text { Mean } \\
\text { Square } \\
\text { Error }\end{array}$ & $\begin{array}{c}\text { Actual } \\
\text { Error }\end{array}$ \\
\hline Godrej & 888.6530 & 0.3345 & 0.079 \\
\hline Finolex Cables & 230.5479 & 0.1999 & 0.123 \\
\hline $\begin{array}{c}\text { TV Today } \\
\text { Networks }\end{array}$ & 250.8452 & 0.1385 & 0.16 \\
\hline Goodyear India & 543.278 & 0.4106 & 0.151 \\
\hline Blue Star & 288.9942 & 0.1657 & 0.128 \\
\hline
\end{tabular}

The time-series plots for different stocks falling under the above mentioned classes were tested for closed loop configuration and closed loop configuration with input delays to analyze the optimum conditions and parameters for the network to work with the highest level of efficiency.

The output given by the network is shown as: The actual price of the stock was 79.50Rs.

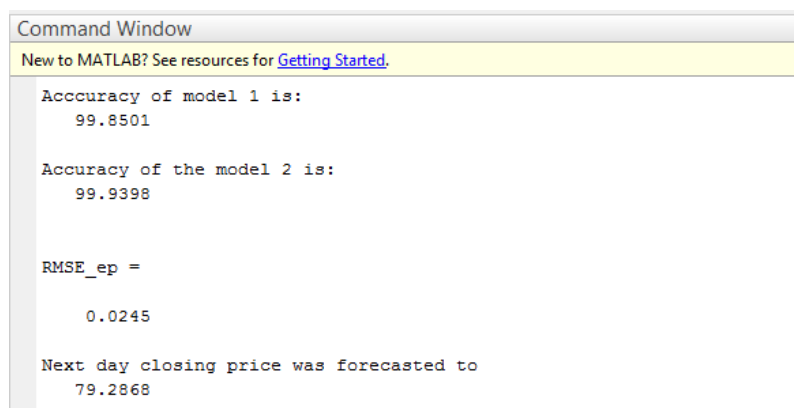

Fig 6. Output of the network

The different time series plots obtained for some of the above mentioned stocks are shown below:

Time series plots for Reliance Communications:-

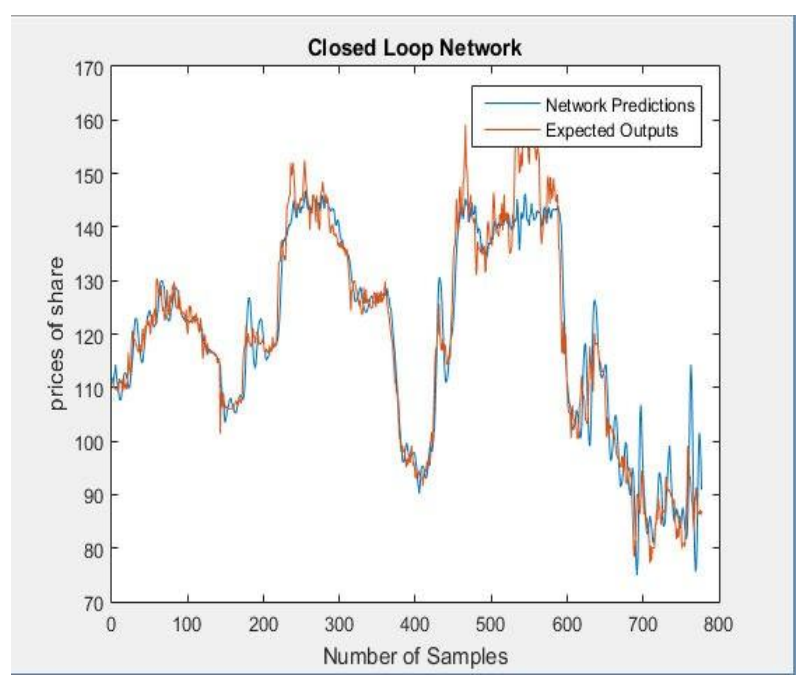

Fig 7 . Time series plot for Reliance Communications for a closed loop network with input delays 


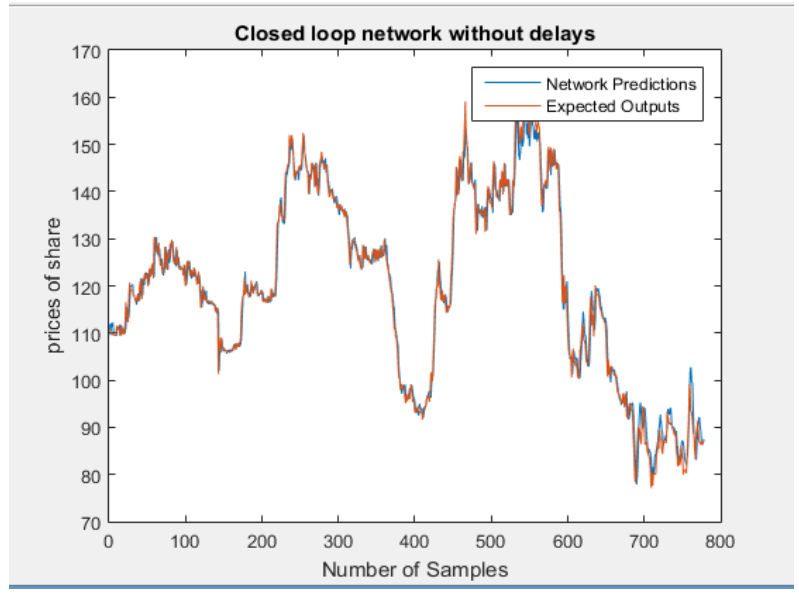

Fig 8. Time series plot for Reliance Communications for a closed loop network without input delays

Time Series Plots for RadicoKhetan :

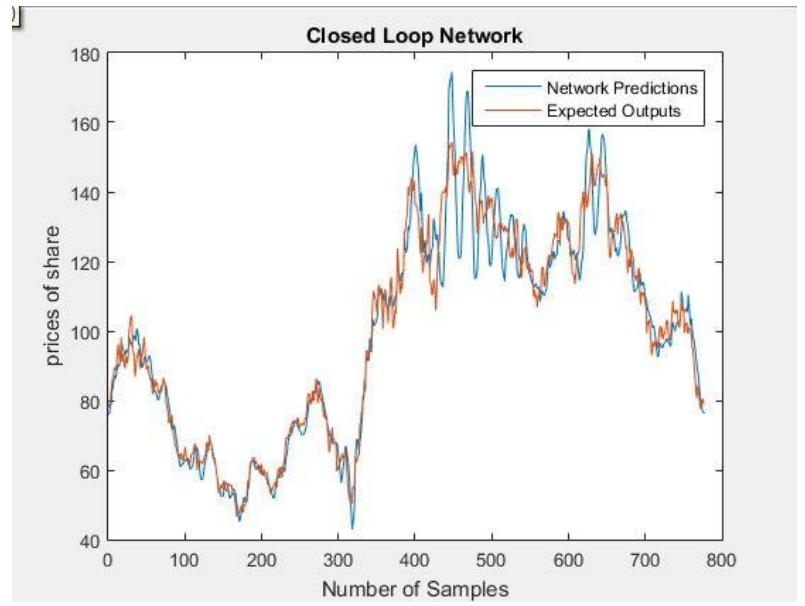

Fig 9. Time series plot for RadicoKhetan for a closed loop network with input delays

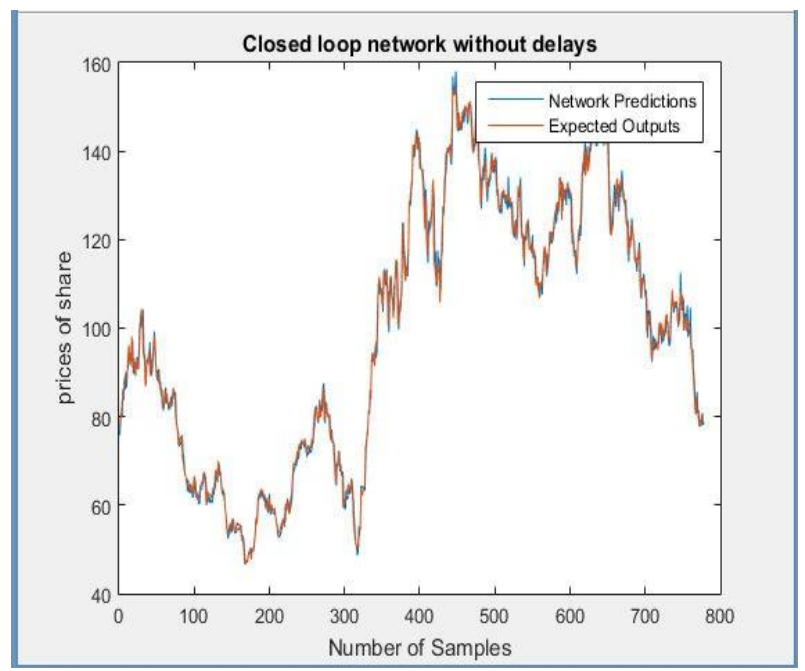

Fig 10. Time series plot for RadicoKhetan for a closed loop network without input delays

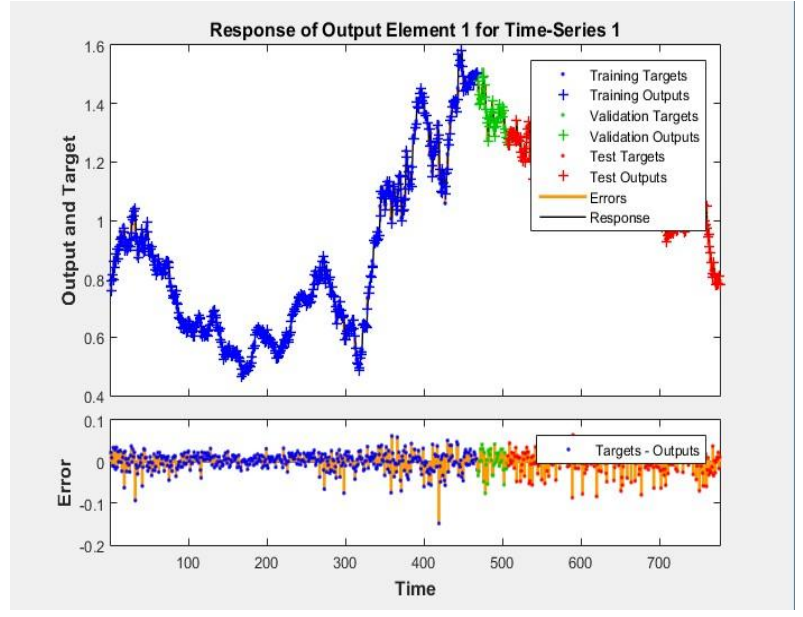

Fig 11.Time series response for the output element

\section{CONCLUSION}

While Stock market prediction has been implemented using neural network algorithms such as the Back-propagation or Radial Basis Functions, the NARX neural network model results in a better accuracy. The ability of the NARX neural network to incorporate time delays results in better computation of stock values based on time series feedback. Implementation of NARX neural network in closed loop form without input delays results in a lower error margin than closed loop with input delay designs and thus is the optimum configuration to ensure better performance of the model. The proposed model has a high degree of prediction for stable and moderately stable stocks. However, highly volatile stocks suffer from minor error fluctuations but the overall accuracy of the model is under the optimum threshold level. . The model also does not incorporate unpredictability in political and economical scenarios and any such sudden events that significantly impact the stock market. Hence, a future model may consider introducing a Gaussian Probability Distribution function to represent the probability of any such event taking place which will have an impact on the stocks.

\section{FUTURE SCOPES}

The proposed model can be incorporated into complex narxnet models which can be used to predict the closing price of a stock over a range of time period or can be used to predict the opening as well as closing price of the stock with precision. Also the accuracy of the model can be further increased by allowing the model to analyze and comprehend the trend of the three exogenous inputs. Also we can incorporate a fuzzy system through which ambiguities in stock Fig 10. Time series plot for RadicoKhetan for a closed loop network without input delays prices during political and economical crisis can be predicted with a very small amount of error.

\section{REFERENCES}

[1] AbhishekKar (Y8021), Department of Computer Science and Engineering, IIT Kanpur, "Stock Prediction using Artificial Neural Networks".

[2] Selvan Simon, ArunRaoot, International Journal on Soft Computing (IJSC), Vol.3 No.2, May 2012 ,"Accuracy Driven Artificial Neural Networks in Stock Market Prediction".

[3] MarijanaZekic, University of JosipJurajStrossmayer in Osijek, "Neural Network Applications in Stock Market Predictions - A Methodolgy Analysis". 
[4] ParasharChandrashekharSoman, a thesis submitted to the Graduate School- New Brunswick Rutgers ,The State University of New Jersey, October 2008, "An Adaptive NARX Neural Network Approach for Financial Time Series Prediction".

[5] "Application of artificial neural network for the prediction of stock market returns:The case of Japanese stock market", Chaos,Solitons and Fractals, Volume 85,April 2016, Mingyue Qui, Yu Song, Fumio Akagi, Department of Systems Management, Fukuoka Institute of Technology.

[6] Images from google, matlab.org.

[7] Bhagwant Chauhan, UmeshBidave, AjitGangathade, SachinKele, International Journal of Computer Science and Information Technologies, Vol 5(1),2014,904-907, "Stock Market Prediction Using Artificial Neural Networks".

[8] Amin Hedayati Moghaddam, Moein Hedayati Moghaddam, "Journal of Economics, Finance and Administrative Science", Science 21 (2016)89-93, "Stock market index prediction using artificial neural network".
[9] AroshineMunasinghe,DajanaVlajic, Royal Institute of Technology DD143X, Bachelor's Thesis in Computer Science, Thesis Supervisor: Pawel Herman, June 2015, "Stock market prediction using artificial neural networks".

[10] "Neural Network Applications in Stock Market Predictions- A Methodology Analysis", MarijanaZekic, MS, University of JosipJurajStrossmayer in Osijek.

[11] Xiaohua Wang, P.K.H. Phua, "Stock Market Prediction using neural networks: Does trading volume help in short-term prediction", Neural Networks, 2003,Proceedings of the International Joint Conference on Neural Networks.

[12] AmirhosseinGhaznavi, Mohammad Aliyari, Mohammad Reza Mohammadi, International Research Journal of Applied and Basic Sciences,ISSN:2251-838X, Vol 10,2016,"Predicting Stock Price Changes of Tehran Artmis Company Using Radial Basis Function Neural Networks".

[13] HakobGrigoryan. Bucharest University of Economic Studies, Buchrest, Romania, "Stock Market Prediction using Artificial Neural Networks, Case Study of TAL1T, Nasdaq OMX Baltic Stock. 